\title{
Laryngeal split and rib cartilage interpositional grafting: Treatment option for glottic/subglottic stenosis in adults
}

\author{
Ricardo Mingarini Terra, MD, Hélio Minamoto, MD, Felipe Carneiro, MD, Paulo Manuel Pego-Fernandes, MD,
} and Fábio Biscegli Jatene, MD

\begin{abstract}
Objectives: Severe glottic/subglottic stenosis (complex laryngotracheal stenosis) is a rare but challenging complication of endotracheal intubation. Laryngotracheal reconstruction with cartilage graft and an intralaryngeal stent is a procedure described for complex laryngotracheal stenosis management in children; however, for adults, few options remain. Our aim was to analyze the results of laryngotracheal reconstruction as a treatment for complex laryngotracheal stenosis in adults, considering postoperative and long-term outcome.
\end{abstract}

\begin{abstract}
Methods: Laryngotracheal reconstruction (laryngeal split with anterior and posterior interposition of a rib cartilage graft) has been used in our institution to manage glottic/subglottic stenosis restricted to the larynx; laryngotracheal reconstruction associated with cricotracheal resection has been used to treat glottic/subglottic/upper tracheal stenosis (extending beyond the second tracheal ring). A retrospective study was conducted, including all patients with complex laryngotracheal stenosis treated surgically in our institution from January of 2002 until December of 2005.
\end{abstract}

Results: Twenty patients (10 male and 10 female patients; average age, 36.13 years; age range, $18-54$ years) were included. There were no deaths, and the postoperative complications were as follows: dysphonia, $25 \%$; subcutaneous emphysema, 10\%; tracheocutaneous fistula, 20\%; wound infection, 15\%; and bleeding, 5.0\%. Eighty percent of the patients were completely decannulated after a mean of 23.4 months of follow-up (range, 4-55 months).

Conclusions: Laryngeal split with anterior and posterior cartilage graft interposition as an isolated procedure or associated with a cricotracheal resection is a feasible and low-morbidity alternative for complex laryngotracheal stenosis treatment.

Laryngotracheal stenosis (LTS) is an important cause of airway obstruction, and its most common cause is endotracheal intubation. Different surgical and endoscopic techniques have been used to deal with this distressing complication, but partial cricoid cartilage resection with primary thyrotracheal anastomosis (cricotracheal resection [CTR]) is considered the procedure of choice by most airway surgeons when the stenosis is limited to the subglottis and upper trachea. ${ }^{1-3}$ However, when the stenosis is close $(<5 \mathrm{~mm})$ or involves the vocal cords (high subglottic or glottic/subglottic stenosis), CTR alone is not applicable once the glottic component is left unsolved, and the more appropriate approach for these cases is controversial. In pediatric populations laryngotracheal reconstruction (laryngeal split with rib cartilage interposition) is performed in cases of glottic/subglottic stenosis, ${ }^{4-8}$ but in adults there are not enough data published to support this approach. For this reason, our aim was evaluate the LTR technique in the treatment of glottic/subglottic stenosis caused by orotracheal intubation in adult patients,

From the Division of Thoracic Surgery, Hospital das Clínicas, University of São Paulo Medical School, São Paulo, Brazil.

Received for publication April 3, 2008; revisions received June 29, 2008; accepted for publication Aug 20, 2008.

Address for reprints: Ricardo M. Terra, MD, Al. Fernao Cardim, 161 ap. 61-Jardim Paulista, CEP: 01403-020 São Paulo, Brazil (E-mail: rmterra@uol.com.br).

J Thorac Cardiovasc Surg 2009;137:818-23

$0022-5223 / \$ 36.00$

Copyright (c) 2009 by The American Association for Thoracic Surgery doi:10.1016/j.jtcvs.2008.08.035 considering the characteristics of the treated stenosis, the surgical procedures performed, and postoperative outcomes and complications.

\section{MATERIALS AND METHODS \\ Patients}

Our facility is a tertiary care hospital and a national reference center for surgical airway disorders. Each week we see about 20 patients with airway disorders, most of them with postintubation stenosis. All patients suspected to have an LTS undergo a flexible bronchoscopy and a neck and chest computed tomographic (CT) scan. These procedures allow us to confirm the diagnosis and evaluate stenosis anatomy, as well as laryngeal motility. Relevant anatomic characteristics are stenosis level and obstruction grade (the last classified according to the Cotton grading system described in Table $1^{9}$ ). Stenosis level is a crucial issue, and all patients are classified depending on compromised airway anatomic structures as follows: tracheal, subglottic, or glottic/subglottic (subglottic stenosis associated with glottic stenosis). In this study were included all patients with a severe (Cotton grade III/IV) glottic/subglottic or high subglottic ( $<5 \mathrm{~mm}$ from the vocal cords) stenosis treated with open surgical intervention in our institution from January of 2002 until December of 2005. A retrospective review of medical records was done, looking for the following information about each patient: sex, age, stenosis morphologic features, history of previous procedures, operation performed, period of time until complete decannulation, and immediate and long-term complications. This study was approved by our institutional ethics board.

Twenty-two patients with complex postintubation LTS were treated in our institution during the study period. Two of them were excluded from the analysis: one because we found that he had a different stenosis cause (Wegener disease instead of postintubation stenosis) and the other because an incomplete procedure was performed (only an anterior laryngofissure 


\section{Abbreviations and Acronyms \\ $\mathrm{CT}=$ computed tomographic \\ $\mathrm{CTR}=$ cricotracheal resection \\ LTS $=$ laryngotracheal stenosis}

with no costal cartilage graft placement). Fifty percent of the 20 patients were men ( 10 male subjects), and the other $50 \%$ were women ( 10 female subjects); the mean age was 36.13 years (range, 18-54 years). Previous procedures were tracheostomy only $(\mathrm{n}=10)$, laryngofissure $(\mathrm{n}=5)$, CTR $(n=2)$, dilatation and T-tube stenting $(n=2)$, and dilatation only $(n=1)$, all of them performed elsewhere. Only 1 patient was not tracheostomized at the time of the operation. The luminal obstruction was Cotton grade III $(n=5)$ or IV $(n=15)$ stenosis. In 14 patients the glottic/subglottic stenosis was restricted to the larynx, and in 6 patients glottic and subglottic stenosis extended beyond the second tracheal ring.

\section{Surgical Technique}

Patients considered for surgical treatment had an extensive clinical evaluation before surgical intervention. These patients frequently have serious comorbidities that might even preclude open surgical intervention. Diabetes, hypertension, coronary disease, and other associated diseases were addressed when present, and the procedure was reconsidered after clinical compensation. For patients with trauma, all neurologic, plastic, or orthopedic procedures were performed before airway reconstruction. The appropriate procedure for each patient was chosen according to stenosis level. We routinely perform stenosis resection and end-to-end anastomosis for isolated tracheal stenosis and partial cricoid resection and thyrotracheal anastomosis for simple subglottic stenosis. For glottic/ subglottic stenosis restricted to the larynx and first tracheal ring, we perform a standard laryngotracheal reconstruction, as described below. For glottic/subglottic/upper tracheal stenosis (beyond the second tracheal ring), laryngotracheal reconstruction associated with a CTR is performed as described below. When the upper trachea is also affected, an associated CTR is mandatory because standard laryngotracheal reconstruction resolves only the laryngeal portion of the stenosis and does not correct the tracheal disease.

Standard laryngotracheal reconstruction. General anesthesia was induced, and the patients were ventilated through the previous tracheostomy. The only patient who had no former tracheostomy underwent rigid bronchoscopy and airway dilatation before orotracheal intubation. A horizontal anterior neck incision 3 to $4 \mathrm{~cm}$ above the sternal notch was made, and strap muscles were separated in the midline. The larynx was exposed, and a midline longitudinal incision, including thyroid and cricoid cartilages (Figure 1, $A$ ), was performed, and both edges were retracted. With the airway exposed (Figure 1,B), a midline incision at the posterior cricoid plate was made from the cricoid-trachea transition up to the arytenoids. The posterior opening must be particularly careful; it can be rather difficult because of stenotic fibrous tissue or adhesions to the anterior esophageal wall.

After the airway was fully opened anteriorly and posteriorly, a costal cartilage was resected for grafting. A horizontal incision was performed

TABLE 1. Cotton endoscopic grading system for subglottic stenosis

\begin{tabular}{lc}
\hline Grade & Lumen obstruction (\%) \\
\hline I & $0 \%-50 \%$ \\
II & $51 \%-70 \%$ \\
III & $71 \%-99 \%$ \\
IV & No visible lumen \\
\hline
\end{tabular}
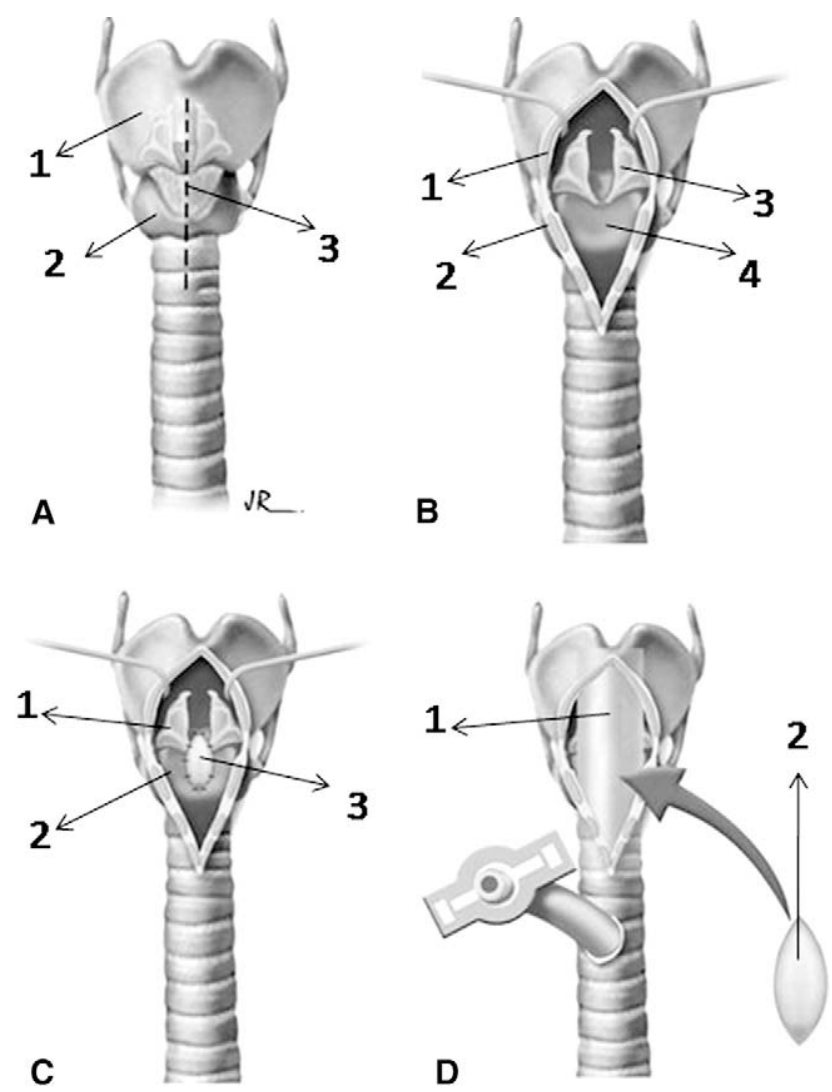

FIGURE 1. Standard laryngotracheal reconstruction. A, Laryngotracheal anterior exposure: 1 , thyroid cartilage; 2 , cricoid cartilage; 3 , incision site to perform the anterior laryngeal split. B, Airway exposure after anterior laryngeal split: 1 , thyroid cartilage split and retracted; 2 , cricoid cartilage split and retracted; 3 , arytenoids; 4 , posterior cricoid plate, exposed and ready to be split. C, Posterior larynx grafting after posterior cricoid split: 1 , arytenoids; 2 , posterior cricoid plate already opened; 3 , rib cartilage graft filling the posterior groove. D, Laryngeal stent and anterior grafting: 1 , solid stent inside the airway; 2 , rib cartilage graft prepared to fill the anterior defect.

in the anterior chest wall over the second costal cartilage that was dissected in a subperichondrial manner and fully resected. The costal cartilage graft was 3 to $4 \mathrm{~cm}$ in length, 1 to $2 \mathrm{~cm}$ in width, and $1 \mathrm{~cm}$ thick; the dimensions varied according to the patient's anthropometry. The resected cartilage was divided longitudinally in 2 symmetric pieces (with the same dimensions of the original but thinner), and both were shaped into an elliptic configuration with a scalpel or a drill.

One of the prepared cartilage grafts was placed in the posterior groove to keep it open and thus enlarge the posterior cricoid surface. PDS 4-0 sutures (Ethicon, Inc, Somerville, NJ) were used to fix the graft to the posterior cartilage edges, thus avoiding extrusion, and no mucosal graft was used to cover it (Figure 1,C). A solid laryngeal stent was positioned inside the airway (Figure 1,D). Most times the previous tracheostomy was kept, but if there was no previous tracheostomy or if it was in contact with the anterior cricoid arch, a new tracheostomy was performed in the second or third tracheal rings. Then the other cartilage graft was placed in the anterior groove, and PDS 4-0 sutures were used to fix the graft to the larynx (Figure 1,D). After that, the wound is closed, and the patient leaves the operating room while breathing spontaneously through the tracheostomy. Drains were not routinely used. 
Laryngotracheal reconstruction associated with CTR. Anesthesia, airway approach, neck incision, and anterior laryngeal split were performed as previously described for standard laryngotracheal reconstruction. Then a CTR similar to the procedure described and popularized by both Pearson and Grillo ${ }^{1}$ was performed. The anterior cricoid arch was resected bilaterally until the cricothyroid joint. Posteriorly, the upper resection margin was the transition between the cricoid and trachea. The inferior resection margin was the end of the tracheal portion of the stenosis and should contain a full-sized healthy tracheal ring. The trachea was mobilized anteriorly and posteriorly, keeping dissection as close as possible to the trachea in the subperichondrial plane to preserve the recurrent laryngeal nerves. During the CTR, we tried to leave a posterior wall flap. After the resection of the anterior cricoid arch and the stenotic portion of the trachea, the posterior subglottic scar was excised, leaving an edge of healthy mucosa below the arytenoids. The patient was ventilated through an orotracheal tube inserted in the distal trachea.

At that moment, the larynx and remnant trachea were separated, and the airway was fully exposed (Figure 2, A). The posterior cricoid lamina was split in the midline from the inferior border up to the interarytenoid ligament. A second costal cartilage was resected and prepared as previously described. One of the grafts was placed in the posterior groove and attached to the cricoid with PDS 4-0 sutures (Figure 2, B). An anastomosis between the posterior tracheal wall flap and the edge of the arytenoid mucosa was performed, covering the posterior cartilage graft (Figure 2, C). Laterally, Vycril 3-0 sutures (Ethicon, Inc) were placed in the trachea and thyroid cartilage anteriorly to the cricothyroid joint. A tracheostomy was fashioned in the healthy lower trachea. A solid laryngeal stent was positioned, and the anterior defect was covered with the other piece of costal cartilage graft fixed to thyroid cartilage with 4-0 PDS sutures (Figure 2, D).

\section{Follow-up}

In all cases we left a solid laryngeal stent that was switched to a T-tube trough an endoscopic procedure after 6 to 10 weeks, allowing normal airway re-establishment.

Every 6 months, a new endoscopic procedure was performed to evaluate the airway and possible decannulation. Decannulation is the most important criterion for success in LTS therapy. We considered decannulation successful when stenting was no longer needed and patients had no or mild dyspnea (grade I or II) at least 3 months after T-tube decannulation. We considered the procedure a failure when stent withdrawal was not possible after 36 months or if a new stenting or surgical procedure (eg, tracheostomy) was required after decannulation.

Figure 3 shows preoperative CT scans and a postoperative CT scan and bronchoscopy of a successfully treated patient which had a severe glottic/ subglottic stenosis. It is interesting to note the anterior and posterior laryngeal defects, which were filled with cartilage grafts, in the CT scan and the overgrowth of mucosa over the cartilage graft in the bronchoscopy.

\section{RESULTS}

All patients were breathing spontaneously after leaving the operating room. No major complications occurred during the surgical procedures, and only 1 patient required a chest tube because of pneumothorax caused by accidental pleural division during costal cartilage dissection. The tube was kept in place until the second postoperative day.

The main results are summarized in Table 2. There was no mortality in the studied group. Immediate complications included wound infections in $3(15 \%)$ patients, which were treated with broad-spectrum antibiotics; in one of them it was also necessary to use subcutaneous abscess drainage. Subcutaneous emphysema was present in $2(10 \%)$ patients,
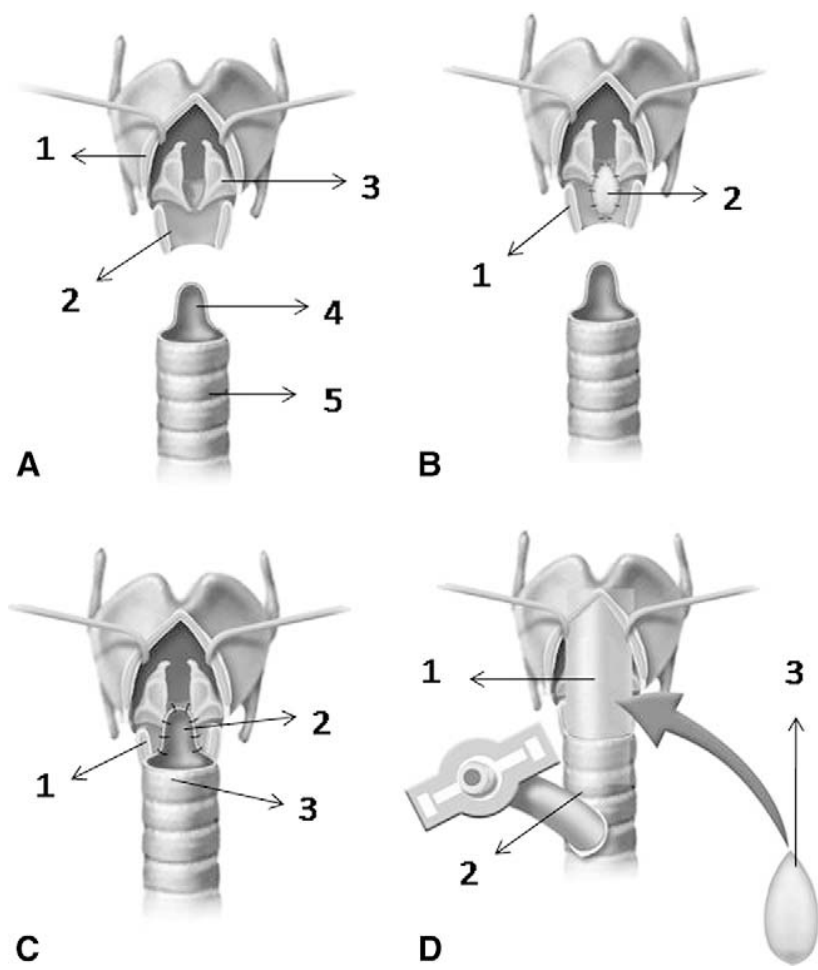

C

FIGURE 2. Laryngotracheal reconstruction associated with cricotracheal resection. A, Larynx apart from the trachea, anterior laryngeal split and cricotracheal resection already performed: 1 , thyroid cartilage split and retracted; 2, posterior cricoid (anterior arch already resected); 3, arytenoid cartilage; 4 , tracheal posterior wall flap; 5 , remaining healthy trachea. B, Cartilage grafting after posterior cricoid split: 1 , posterior cricoid already split; 2 , rib cartilage graft filling the posterior cricoid plate groove. C, Posterolateral cricotracheal anastomosis hiding the posterior cartilage graft: 1 , posterior cricoid cartilage; 2 , posterior tracheal wall flap sutured to posterior cricoid remnant mucosa; 3 , healthy trachea, posterolateral anastomosis with the larynx already completed. D, Laryngeal stent and anterior grafting: 1 , laryngeal stent; 2 , trachea, tracheostomy already performed; 3 , rib cartilage graft prepared to fill the anterior defect.

which coursed with spontaneous regression. Bleeding was present in $1(5.0 \%)$ patient, who required operating room exploration to promote hemostasis and bleeding control of an anterior jugular vein. Significant dysphonia (low pitch, hoarseness, and reduced vocal intensity evaluated by an otolaryngologist) occurred in $5(25 \%)$ patients. Postoperative laryngoscopy excluded vocal fold palsy in these patients but showed significant anatomic alterations as vocal fold asymmetry and misapproximation during closure. Three of them improved with phonotherapy and observation; however, the other 2 had long-term bad voice quality. Persistent tracheocutaneous fistulas after decannulation occurred in $4(20 \%)$ patients (all of them had a grade IV stenosis before the repair), and after a 30-day observation period, all these patients underwent surgical fistula closure in our outpatient clinic.

During the study period, $80 \%$ of the patients $(n=16)$ were successfully decannulated. They remained for 

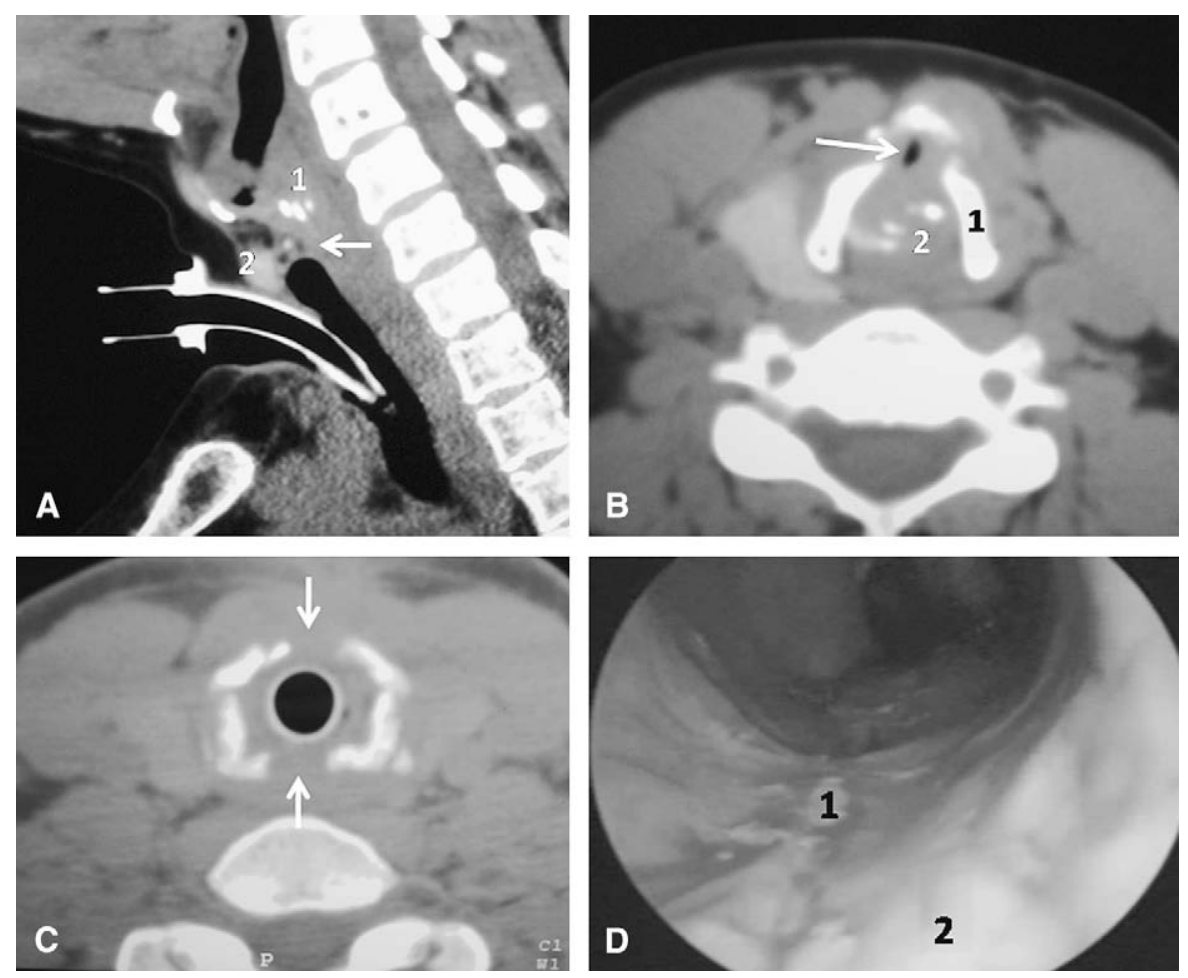

FIGURE 3. A, Saggital cervical computed tomographic scan showing glottic/subglottic stenosis (arrow): 1, arytenoid cartilage; 2 , anterior cricoid arch. B, Axial cervical computed tomographic scan showing severe glottic stenosis (arrow): 1, thyroid cartilage; 2, asymmetric arytenoids. C, Postoperative neck computed tomographic scan showing cricoid cartilage with anterior and posterior discontinuity (arrows) caused by the laryngeal split; the patient still has the T-tube placed. D, Postoperative endoscopy showing an adequate laryngeal lumen: 1 , overgrowth of the mucosa over the cartilage graft; 2 , arytenoid cartilage.

58.4 days on average (range, 14-137 days) with solid laryngeal stent and 22.5 months on average (range, 2-34 months) with T-tube stenting. The mean time until complete decannulation was 23.4 months (range, 4-55 months). None of these patients required any additional open surgical or endoscopic procedures, and all of them remained free of symptoms during a postdecannulation mean follow-up of 28 months.

In $4(20 \%)$ patients the treatment failed. One of them had postoperative malacia, and permanent stenting was necessary to maintain a good airway. The other 3 patients had stenosis recurrence and needed new interventions 2, 4, and 6 weeks after decannulation. Two of them were eventually tracheostomized, and the other patient underwent an endoscopic procedure for a silicone endoprosthesis placement. They are still followed up in our ambulatory department. Postoperative complications observed in other repair techniques, such as bronchoaspiration, or even significant deglutition disturbances did not occur in our series.

\section{DISCUSSION}

Treating LTS is a challenge, but treating a glottic/subglottic stenosis is an even bigger challenge. Most series addressing LTS treatment exclude patients with associated glottic stenosis. ${ }^{10}$ However, these patients do exist, and their management is not easy. Maddaus and colleagues ${ }^{11}$ proposed a combined procedure in which an anterior laryngotomy is performed after CTR, and glottic stenosis is managed; no graft interposition is used. In this procedure the patient

TABLE 2. Outcomes and complications

\begin{tabular}{lccc}
\hline & LTR & LTR/CTR & Total \\
\hline $\mathrm{n}$ & 14 & 6 & 20 \\
Complications & & & \\
$\quad$ Infection & 2 & 1 & $3(15 \%)$ \\
$\quad$ Subcutaneous emphysema & 1 & 1 & $2(10 \%)$ \\
$\quad$ Bleeding & 1 & 0 & $1(5 \%)$ \\
$\quad$ Dysphonia & 4 & 1 & $5(25 \%)$ \\
$\quad$ Tracheocutaneous fistula & 3 & 1 & $4(20 \%)$ \\
Time & & & \\
$\quad$ Laryngeal stent (d) & 57.8 & 60.2 & $58.4(14-134)$ \\
$\quad$ T-tube (mo) & 23.5 & 21.2 & $22.5(2-49)$ \\
$\quad$ Until total decannulation (mo) & 25.2 & 20.3 & $23.4(4-55)$ \\
$\quad$ Follow-up after & 29 & 27 & 28 \\
$\quad$ decannulation (mo) & & & \\
Success rate & & & \\
$\quad$ Decannulation & 11 & 5 & $16(80 \%)$ \\
\hline
\end{tabular}

$L T R$, Standard laryngotracheal reconstruction; $L T R / C T R$, laryngotracheal reconstruction associated with cricotracheal resection. 
leaves the operating room with a T-tube in place. The authors reported satisfactory to good results in $86 \%$ of 15 patients who underwent this procedure. However, neither further publications addressing this technique nor late results were found in the literature.

In the pediatric population LTR has been used by many authors to deal with LTS successfully, even when they are complex cases..$^{4-7,12}$ A $96 \%$ decannulation rate was reported with this approach, ${ }^{4}$ although when individualizing patients according to stenosis grade, we observed worse results. Good outcome was also reported with this technique in difficult-to-manage patients, such as patients with Cotton grade III and IV stenosis. ${ }^{5}$ However, none of these analysis have addressed the specific glottic/subglottic stenosis population or identified them in their series. Regarding the adult population, LTR was common in the past, but it was left behind after the establishment of the CTR technique, and few reports have been published since then.

As also seen in the literature, ${ }^{1-3}$ we consider CTR the best surgical approach for patients with LTS. However, the glottic component was always a concern for this select group of patients with glottic/subglottic stenosis. Because we are a referral center, more and more complex cases have appeared, many of them failed cases treated in smaller centers. Conceptually, we believe that the glottic and subglottic component should be dealt with at the same procedure, and therefore we started to perform LTR for these complex cases. Encouraged by Maddaus and colleagues ${ }^{11}$ and experience reported elsewhere, we also started, when possible, performing the previously described associated procedure when the tracheal stenotic segment extended beyond the second tracheal ring.

We consider temporary stenting mandatory. Although some studies in children have shown good results for procedures avoiding postoperative stenting, ${ }^{13}$ the only article reporting this approach in adults showed fair results. ${ }^{14}$ Because of this lack of evidence and because of the complexity of treated cases, we preferred to leave a solid laryngeal stent in all patients, which was changed to a T-tube in 8 weeks. Both solid stent change and T-tube weaning are also controversial issues. In the literature we found nothing but personal experiences or preferences. We arbitrarily determined that we would switch the solid stent to a T-tube in about 8 weeks and that this T-tube would be re-evaluated every 6 months. The criteria for T-tube complete decannulation was subjective and based on airway features (eg, inflammation, malacia, and granulation tissue). At the beginning, patients remained with a T-tube for up to 3 years. With accumulated experience, we noticed that this time could be shortened. We had a patient who had a T-tube in place for 4 months only and had an excellent result. Decannulation time and some technique improvements are the subject of a prospective study we are carrying out about this management strategy in patients with complex LTS.
The wound infection rate was quite high in our patients $(15 \%)$ when compared with other similar procedures. That is probably due to the high proportion of chronically tracheostomized patients (all but one). Long-term tracheostomies are frequently colonized and infected, and thus they might increase the intraoperative contamination despite adequate preoperative care and antibiotics. We do not routinely use drains after laryngotracheal operations; however, the high infection rate found in this series made us reconsider their use in this specific subset of patients because a better subcutaneous drainage might prevent some of the infection cases.

The most frequent complication was dysphonia, which is rarely seen in CTR (and is frequently associated with recurrent nerve lesions and vocal fold paralysis). LTR can cause laryngeal functional impairment, and other series had $44 \%$ and $52 \%$ dysphonia rates, respectively. ${ }^{15,16}$ It is important to note that $75 \%$ of our patients had complete laryngeal occlusion (Cotton grade IV) before the repair, and therefore they had very low or no voice at all for several months or even years, which has a great effect in vocal rehabilitation. A complication observed in our series that is rarely reported in other series is tracheocutaneous fistula, a consequence of prolonged tracheostomy. Maybe if we were able to shorten the decannulation time, this complication would be less frequent.

Our decannulation rate was $80 \%$, which is less than that for CTR in patients with simple LTS (only a $2.5 \%$ failure rate observed in some series ${ }^{1,2}$ ) but quite acceptable for such a difficult-to-manage population; Maddaus and colleagues ${ }^{11}$ reported $86 \%$ success in a similar subset of patients. Most of the failed cases $(75 \%)$ were due to stenosis recurrence and were diagnosed until 6 weeks after decannulation. We do not know whether they had had their T-tubes in place for a longer period; the course would have been the same (although all had the T-tubes in place $>12$ months). It is also interesting to note that all patients experiencing failure were severely obese (body mass index [BMI] > 35). Unfortunately, the small number of patients precluded an analysis of risk factors for recurrence and complications.

The anterior laryngofissure involves anterior glottic commissure opening, and therefore it might cause deglutition disturbances and bronchoaspiration. Even though this issue was not addressed through specific tests in our population, no clinically relevant symptoms were observed other than mild transitory postoperative dysphagia reported by 2 patients.

Because this is a retrospective study with no control group, only weak evidence can be drawn from it. In this study we had different follow-up times among patients, and therefore we do not know whether we will observe long-term disease control for all patients. A later analysis will show such results. Outcome comparisons between the 2 procedures were not made because they are performed in different patient populations. Because complex LTS is not 
a frequent entity, a randomized controlled study would not be easily performed, and maybe a multi-institutional study should address this population to bring more light to this controversial issue.

With this approach, we had results similar to those already published in the literature $(80 \%$ decannulation rate and low morbidity) but in a very difficult-to-manage population. We still consider time until decannulation too long; however, this will be a subject for the next prospective study. We conclude that laryngotracheal reconstruction, either standard or associated with CTR, is a good alternative for complex LTS treatment.

\section{References}

1. Grillo HC, Mathisen DJ, Wain JC. Laryngotracheal resection and reconstruction for subglottic stenosis. Ann Thorac Surg. 1992;53:54-63.

2. Macchiarini P, Verhoye JP, Chapelier A, Fadel E, Dartevelle P. Partial cricoidectomy with primary thyrotracheal anastomosis for postintubation subglottic stenosis. J Thorac Cardiovasc Surg. 2001;121:68-76.

3. Ashiku SK, Kuzucu A, Grillo HC, Wright CD, Wain JC, Lo B, et al. Idiopathic laryngeal stenosis: effective definitive treatment with laryngotracheal resection. J Thorac Cardiovasc Surg. 2004;127:99-107.

4. Gustafson LM, Hartley BE, Liu JH, Link DT, Chadwell J, Koebbe C, et al. Singlestage laryngotracheal reconstruction in children: a review of 200 cases. Otolaryngol Head Neck Surg. 2000;123:430-4.
5. Schick B, Weidenbecher M, Miller R, Iro H. Experience with laryngotracheal reconstruction in subglottic stenosis in a 30 years time period. Laryngorhinootologie. 2007;86:358-64

6. Hartley BEJ, Cotton RT. Paedriatic airway stenosis: laryngotracheal reconstruction or cricotracheal resection? Clin Otolaryngol. 2000;25:342-9.

7. Agrawal N, Black M, Morrison G. Ten-year review of laryngotracheal reconstruction for paediatric airway stenosis. Int J Pediatr Otorhinolaryngol. 2007;71: 699-703.

8. Walner DL, Stern Y, Cotton RT. Margins of partial cricotracheal resection in children. Laryngoscope. 1999;109:1607-10.

9. Myer CM, O'Connor DM, Cotton RT. Proposed grading system for subglottic stenosis based on endotracheal tube sizes. Ann Otol Rhinol Laryngol. 1994; 103:319-23.

10. Macchiarini P, Chapelier A, Lenot B, Cerrina J, Dartevelle P. Laryngotracheal resection and reconstruction for postintubation subglottic stenosis. Lessons learned. Eur J Cardiothorac Surg. 1993;7:300-5.

11. Maddaus MA, Toth JL, Gullane PJ, Pearson FG. Subglottic tracheal resection and synchronous laryngeal reconstruction. J Thorac Cardiovasc Surg. 1992;104: 1443-50.

12. Cotton RT. Prevention and management of laryngeal stenosis in infants and children. J Pediatr Surg. 1985;20:845-51.

13. Younis RT, Lazar RH. Laryngotracheal reconstruction without stenting. Otolaryngol Head Neck Surg. 1997;116:358-62.

14. Rhee JS, Toohill RJ. Single-stage adult laryngotracheal reconstruction without stenting. Laryngoscope. 2001;111:765-8.

15. MacArthur CJ, Kearns GH, Healy GB. Voice quality after laryngotracheal reconstruction. Arch Otolaryngol Head Neck Surg. 1994;120:641-7.

16. Clary RA, Pengilly A, Bailey M, Jones N, Albert D, Comins J, et al. Analysis of voice outcomes in pediatric patients following surgical procedures for laryngotracheal stenosis. Arch Otolaryngol Head Neck Surg. 1996;122:1189-94. 